\title{
COVID-19: WHAT'S NEXT?
}

\author{
Alfredo Ponce-de-León ${ }^{1}$, María Dolores Niembro-Ortega ${ }^{2}$, and María F. González-Lara ${ }^{1 *}$ \\ ${ }^{1}$ Department of Infectious Diseases and ${ }^{2}$ Directorate of Medicine, Instituto Nacional de Ciencias Médicas y Nutrición \\ Salvador Zubirán, Mexico City, Mexico
}

\begin{abstract}
Since December 2019, when severe acute respiratory syndrome coronavirus 2 emerged in Wuhan, China, this virus and the resulting disease, coronavirus disease (COVID-19), has spread worldwide. What has occurred in this year and a half goes beyond anything we have dealt with, as humankind, in the past two centuries, perhaps obscured only by war. An incredible number of articles, whether scientific or in the press, have been published, making it impossible to discern between what is biological and what is social in nature. Here, we aim to reflect on the basic structure of the virus and associate its behavior to that of determining factors of the human condition that may be modifiable soon. Needless to say, we find our effort clearly incomplete, and that both scientific and social aspects regarding COVID-19 or any other pandemic encountered in the future, will be constantly changing, from their beginning to their end. (REV INVEST CLIN. 2021;73(5):329-34)
\end{abstract}

Key words: Coronavirus disease. Severe acute respiratory syndrome coronavirus 2 variants. Vaccines. Nanotechnology. Artificial intelligence.

If anything could be said related to the pandemic ordeal, it is that to understand its effect beginning with the virus and translating into humans, one should not only look at the pieces of the puzzle separately, but should make the effort to comprehend the complexity of the intrinsic dynamics of the encounter and, then, place them together into a logical matrix. This approach will ultimately integrate the strategies of the different countries that are critical for the prevention and promotion of health from a global perspective $^{1}$. However, a basic understanding of this initial encounter has given us the opportunity to decipher why severe acute respiratory syndrome coronavirus 2
(SARS-CoV-2) has created such havoc when compared to other respiratory viruses.

Based on the structural properties of its membrane and nucleocapsid, it seems that the virus is highly resistant to conditions outside the human body and also in body fluids, characteristics that may favor alternative transmission routes. As soon as the pandemic started, it was recognized that the virus enters the lung alveolar type 1 cells through angiotensin-converting enzyme-2 (ACE2) receptors ${ }^{2}$. However, this interaction is different from that between SARS-CoV and the same receptor: the affinity of SARS-CoV-2 spike
*Corresponding author:

María F. González-Lara

E-mail: fergonla@gmail.com
Received for publication: 06-07-2021

Approved for publication: 10-07-2021

DOI: $10.24875 / R I C .21000362$

0034-8376 / (c) 2021 Revista de Investigación Clínica. Published by Permanyer. This is an open access article under the CC BY-NC-ND license (http://creativecommons.org/licenses/by-nc-nd/4.0/). 
protein $(S)$ for the human ACE2 is 10-20-fold higher than that of the SARS-CoV spike protein ${ }^{3}$. This is important since it may explain why SARS-CoV-2 enters lung cells at super efficiency. As Ortega suggests ${ }^{4}$, these higher values of affinity might be related to the increased dynamics of the infection, and the rapid spread observed for this virus. Of note, viral entry is facilitated after fusion of S2 (the membrane fusion subunit of S) with ACE2 through proteolysis of TMPRSS2, the transmembrane serine protease. This widely known concept of facilitated entry may be shadowed by one of the many differences between the $\beta-\mathrm{CoVs}$, that is, a polybasic cleavage site at the junction of the S1-S2 subunits, which is subject to furin and other proteases $^{5}$. This is a critical difference between this virus and other coronaviruses, mainly because furinlike proteases are ubiquitous and could participate in expanding cell and tissue tropism, increasing transmissibility, and/or altering pathogenicity ${ }^{6}$. Thus, although TMPRSS2 is crucial for the cleavage of the $\mathrm{S}$ subunits, and therefore, fusion and entry into the cells, other minor proteases may also play a role. This is important since TMPRSS2 may as well be targeted by newer potentially pharmacologic agents. Up to now, camostat and nafamostat, both potent inhibitors of TMPRSS2 activity, are being tested in clinical trials.

It is widely accepted that enveloped RNA viruses use extracellular viruses to translocate into other cells. These vesicles, as stated by Altan-Bonnet, "enable the viruses to infect cells in both receptor-dependent and receptor-independent manner and promote viral persistence. They modulate the host immune response, transport populations of viral particles and genomes, increase multiplicities of the ways of viral infection, facilitate cooperative interactions, and enhance the viral replicative fitness.". These particles, which are not identifiable by immune responses, may be used as a cellular transport pathway, similar to undercover agents, and may reappear long after patients have been considered as recovered ${ }^{8}$. We assume that these patients are not infectious, but they are still a great concern from the public health perspective, because there is no definite evidence regarding the pathogenicity of their viruses within this host-dependent environment. Are these patients serving as a mutation factory for the virus in its way to adapt better to humans? This and other questions remain unanswered, as well as the possibility to find other mechanisms to treat the disease.

\section{HUMANS}

As stated previously, ACE2 acts as a receptor for SARS-CoV-2. Therefore, the physiology of ACE2 is essential to understand the different roles this enzyme plays in the clinical manifestations and outcomes of the infection?. It mediates both pro- and anti-inflammatory processes, and the up- and downregulation of these processes has a profound impact on the disease ${ }^{10}$. Of note, the dysregulation of ACE2, along its distinctive expressions in tissues, is diverse among specific comorbid states, which explains why certain conditions have poorer outcomes and somber prognosis.

At present, what the virus does, is still a matter of discussion, but recent studies have shown that nonstructural protein 1 blocks mRNA translation of host cells and blocks mediators of the innate immune response. Interestingly, the virus activates cell kinases in vitro, which hijack host's protein production, but it also leads to cell cycle arrest. Certainly, some drugs may interfere with these viral processes and eventually could be potential treatments for the disease ${ }^{11}$.

Independently of the viral mechanisms of immune escape, it seems clear that the immune response is dysregulated in the severe and critical phases of the coronavirus disease (COVID-19). In fact, the hallmark of the disease in patients who require hospitalization is an initially exaggerated innate immune response, with high levels of pro-inflammatory markers, and if progressive, a suppressed adaptive immune response with low $\mathrm{T}$ - and B-cell counts. In the very few studies that have obtained cells from bronchoalveolar lavages, there is an increase of inflammatory macrophages which express interferon stimulatory genes, thus signaling the progression of lung damage observed in critical patients. However, this interferon response is delayed, perhaps as a result of an induced synthesis of viral mRNAs, which mimic host cell mRNAs, therefore protecting the virus from the host innate immune response, as demonstrated by Viswanathan et al. ${ }^{12}$. Finally, and irrespective of the pro- or anti-inflammatory processes involved in different viral respiratory infections, what is remarkable of SARS-CoV-2 is that the outcome of the disease is more dependent on host factors, such as age and comorbidities. Whether immunophenotypic differences between individuals may predispose them to a more severe clinical course 
is still under study, but as we have recently described, the frequency of CD19+ B cells is increased in severe COVID-19 and the existing relationship of B-cell subset frequencies with clinical and laboratory parameters suggest that these lymphocytes could serve as potential biomarkers and even active participants in the adaptive antiviral response mounted against SARS-CoV- ${ }^{13}$. Thus, it seems that not only a T-cellmediated response is compromised, but there is also a deficient antibody response. In fact, several studies are under way to study the use of intravenous immunoglobulin in severe COVID-19 cases. The main reason for this approach is that neutralizing antibodies for viral spikes are observed within 21 days of infection in mild-to-moderate cases, and a poor outcome is observed when absent. Unfortunately, convalescent plasma studies have failed to show any benefit thus far.

\section{MODIFYING THE INTERACTION BETWEEN THE VIRUS AND HUMAN DISEASE}

A nucleotide analog, remdesivir, has received great attention. Conflicting evidence has predominated over the real value of this repurposed drug. Its effect is on the RNA-dependent RNA polymerase that interferes with its activity and reduces viral synthesis. However, if promptly used, it leads to a rapid reduction in II- 6 levels. Of note, this gradual and rapid descent in II-6 level is observed when dexamethasone is used, as well as tocilizumab ${ }^{14,15}$. Early on, dexamethasone treatment reduced death by one-third as compared with COVID-19 patients who received only standard supportive care treatment ${ }^{16}$. Recently, the combination of remdesivir (an antiviral) and baricitinib (a JAK inhibitor) has shown to be better than remdesivir alone in patients with moderate COVID-19 disease ${ }^{17}$. This is important, since for the $1^{\text {st }}$ time, the combination of an anti-inflammatory drug and antiviral drug has shown a clear benefit in any infectious disease. Combination managements of other infectious diseases are warranted in the future. Therefore, it is important to note that suppression of pro-inflammatory pathways and the inhibition of inflammatory pathways, either using monoclonal antibodies (Mabs) or intracellular signaling inhibition with anti-endocytosis mechanisms (inibs), in combination with direct anti-viral (through inhibition of RdRp or other cysteine proteases) may be the way in which to control the pathogenesis of the infection, but perhaps more importantly, a better way to avoid progression. Similar to what is currently being done with anti-cancer treatment, target-based computational analysis is possibly the best way to detect potential treatments ${ }^{18}$. A rational understanding of the ultrastructure of RdRP has rendered prospective candidates, either for novel drugs or for repurposed ones to treat SARS-CoV-2 infection ${ }^{19}$. In fact, using this computational highthroughput approach, nelfinavir and the parent prodrug MK4482/EIDD-2801 were found most promising among 12,000 compounds and selectively inhibit SARS-CoV-2 replication; MK4482/EIDD-2801 (the nucleoside analog $\mathrm{N}^{4}$-hydroxycytidine $[\mathrm{NHC}]$ ) has shown broad-spectrum anti-viral activity against RNA viruses by causing an error catastrophe in virus replication ${ }^{20}$. Phase 3 clinical trials are underway in ambulatory patients, but initial results have shown promising results in patients with mild and moderate disease, rapidly eliminating viral load, and hopefully stopping transmission (author's personal communication).

\section{STILL... THE PROBLEM IS CONTINUOUS TRANSMISSION, VIRAL ADAPTATION, AND DIAGNOSTICS}

One of the many problems encountered thus far in the pandemic, and perhaps the greater cause of alarm once variants of concern keep spreading with seemingly diverse symptoms when compared to the initial waves, is the prompt and accurate diagnosis of cases. Along the lines of computational drug-targeted analysis, artificial intelligence methods have been applied to COVID-19 pandemic ${ }^{21}$. It seems reasonable that machine learning and deep learning tools will be applied to this pandemic and other medical problems soon. In fact, combinatorial analysis of image techniques and clinical symptoms had a higher sensitivity for COVID diagnosis than experienced thorax CT scan interpretation. This is extremely important in an over-burdened medical center since relying on artificial intelligence will certainly help emergency room and triage zones. At the institute, we have constructed clinical scores that include clinical history aspects as well as simple parameters that may be used in general hospitals and in primary care centers, to predict possible complications and progression to critical stages of the disease ${ }^{22}$. A reappraisal of this 
and other scores will be needed in patients presenting to the triage zones who have been previously vaccinated, and probably better and more accurate diagnostic testing will be needed to detect the newer variants.

\section{VACCINES}

In the past 15 months, we have witnessed an incredibly rapid vaccine development against COVID-19, an achievement based on pre-existing vaccine experience with SARS-CoV, Middle East respiratory syndrome coronavirus, influenza, HIV, Ebola, and Zika. COVID-19 vaccine platforms are diverse, from lipid nanoparticles encapsulated mRNA encoding the spike proteins, to recombinant adenovirus or inactivated SARS-CoV-2, among others, which have been used; the strategy for avoiding infection is common, producing neutralizing antibodies against the receptor binding domain to block viral attachment to ACE2 cell receptors. It is desirable that vaccines also induce production of memory $T$ and $B$ cells, as efficient and long-term protection from SARS-CoV-2 requires innate, humoral, and cellular immunity ${ }^{23}$.

Fortunately, vaccine effectiveness studies after 14 days of the second dose have demonstrated protection in regard to hospitalization, severe diseases, and COVID-19-associated death $>90 \%$ in most of the available platforms. Studies suggest that vaccination reduces asymptomatic as well as symptomatic infection and is also associated with lower viral RNA levels in the upper respiratory tract and thus presumably lower infectivity.

Further evaluation is required to answer questions regarding duration of protection from disease, potential need for and timing of additional booster doses, effectiveness in immunosuppressed populations ${ }^{24}$, the role of pre-existing antibodies against SARS$\mathrm{CoV}-2$, and breakthrough vaccine infections and their association to variants of concern that escape the immune response directed against spike proteins targeted by the original vaccines. Based on reports made to the CDC in the United States, breakthrough infections after vaccination affect $1 \%$ of those fully vaccinated; of them, $10 \%$ were hospitalized and $2 \%$ died. The proportions caused by variants of concern are under study.
RNA viruses are known to have very high rates of mutation, which correlate with virulence modulation and the ability to evolve. A significant challenge in COVID-19 vaccine development is precisely this high frequency of mutations in the SARS-CoV-2 S protein. An ideal COVID-19 vaccine platform must allow easy and rapid accommodation of newly mutated and identified antigens, especially those recently found in variants of concern ${ }^{25}$.

Animal studies using nanoparticles with cytokine binding properties to decrease pro-inflammatory cytokine expression and repaired lung tissue or studies using nanomaterials for carrying anti-inflammatory drugs to calm the local cytokine release syndrome in pneumonic lungs are promising approaches to developing efficient and safe COVID-19 vaccines. It is known that the clinical advantages of using nanotechnology platforms in these vaccines are the increased stability of the vaccine materials for prolonged immunization, improved pharmacokinetics and pharmacodynamics of the antigens, and enhanced immune response.

Among the most important and urgent aspects to be solved are to enable scalable manufacturing and worldwide distribution along with its faster and precise delivery, as big differences in vaccine availability between countries are evident.

\section{SARS-CoV-2 VARIANTS}

As all viruses, SARS-CoV-2 continuously adapts to changing environments through random mutations that result in natural selection. Most mutations are neutral or detrimental to the virus, but some may lead to increased fitness for transmissibility, escape to the host's immune system or antiviral drug resistance. Three nomenclature systems that name and track distinct genetic lineages are in use: The Global Initiative on Sharing all Influenza Data (GISAID), Nexstrain, and Pango. The WHO recommended using labels with letters of the Greek alphabet to simplify the nomenclature. A variant of interest (VOI) is a SARS-CoV-2 isolate that has genome mutations with established or suspected phenotypic implications that cause community transmission or multiple COVID-19 clusters in numerous countries. A variant of concern (VOC) is a $\mathrm{VOI}$ that has been associated with increased 
transmissibility or detrimental change in COVID-19 epidemiology, increased virulence or change in clinical presentation or decreased effectiveness of public health measures, available diagnostics, vaccines, and therapeutics 26,27 .

Amino acid changes within the receptor-binding motif or the amino terminal domain of the spike protein that enables immune evasion by interfering with neutralizing antibodies were detected as early as January 2020 in China and Germany. A G614 variant demonstrated increased replication and transmissibility but not higher risk of hospitalization. Another lineage with a $\mathrm{N} 439 \mathrm{~K}$ substitution which enhances the binding affinity for the ACE2 receptor and reduces antibody binding emerged and circulated in many European countries. The first detected $\mathrm{VOI}$, a Y453 variant carrying several spike mutations, was identified in Denmark. These lineages are hypothesized to have arisen in chronic infections or previously infected individuals 26,28 .

The circulation of several VOC has changed the course of the SARS-CoV-2 pandemic accelerating existing outbreaks in countries with persistent transmission and causing new outbreaks in countries with decreased infections. The Alpha variant (B.1.1.7 lineage) was first identified in September 2020 in the United Kingdom causing an increment in regional infection rates. It has several mutations within the spike protein, increased transmissibility, and it may increase disease severity. Thus far, this variant has not been associated with immune escape. The Beta variant (B.1.351 lineage) was described in South Africa and subsequently identified in other countries. It causes increased transmissibility and contains a mutation in the spike protein, $\mathrm{E} 484 \mathrm{~K}$, which has reduced antibody neutralization. Convalescent plasma has none neutralizing activity against this variant, whereas recipients of mRNA vaccines have lower antibody titers but maintain neutralizing activity. The Gamma variant (P.1 lineage) was described in Brazil in November 2020 and harbors three spike protein mutations that may result in increased transmissibility, including the E484K mutation. The Delta variant (B.1.617.2 lineage) was identified in India in December 2020 and has become one of the most prevalent variants in several countries. This variant has increased transmissibility and hospitalization rate. The Epsilon variant (B 1.427 and B.1.429 lineages) was described in
October 2020 in South California and is associated with increased cell entry and reduced susceptibility to neutralization by convalescent and vaccine recipient plasma $^{26,28,29}$.

VOI and VOC detection requires sequencing using metagenomics and SARS-CoV-2 targeted identification with a phylogenetic approach. The WHO strongly encourages countries to perform sequencing and rapid public sharing of sequences and metadata. Clinical samples must contain high viral loads and low levels of human or bacterial genetic material to maximize sequencing yield, such as nasal or oral swabs or saliva that has been already processed by a molecular diagnostics laboratory. Samples with reverse transcription polymerase chain reaction cycle thresholds of approximately 25-30 are appropriate. Conventional (Sanger) sequencing can be used, and it is most useful for sequencing short fragments of genomes. Next-generation sequencing platforms (Illumina, IonTorrent, and Oxford Nanopore Technologies) are more appropriate for routine whole-genome sequencing and allow sequencing of multiple fragments of the SARS-CoV-2 genome. Repositories such as GISAID have facilitated data sharing and variant detection ${ }^{27}$.

Plasma from participants vaccinated with BNT162b2 (Pfizer-BioNTech) vaccine maintain neutralizing activity against the Alpha, Beta, and Delta variants, although lower titers were found for the latter. Studies using plasma from recipients of the mRNA-1273 (Moderna) vaccine show preserved neutralizing activity against the Alpha and Epsilon variants; although it can neutralize the Beta variant, 6-9-fold reduced titers are seen. The ChAd0x1 nCoV-19/AZD1222 (Oxford University \& AstraZeneca) vaccine induces lower neutralizing antibody titers against the Alpha variant, but similar efficacy. However, it does not reduce infection caused by the Beta variant in South Africa.

The Ad26.COV2.5 (Janssen/Johnson \& Johnson) vaccine reported variable efficacy in geographic regions with $74 \%$ in the United States, $66 \%$ in Brazil, and 52\% in South Africa, although efficacy to prevent severe disease was $>73 \%$ in this country. The NVX-CoV2373 (Novavax) vaccine seems highly effective against the Alpha variant, but reduced efficacy (49.4\%) against the Beta variant was seen ${ }^{28,30}$. 
New variants will continue to emerge, so health public measures of masking and socially distancing are encouraged, particularly where vaccine coverage is suboptimal. Investment in metagenomics and bioinformatics training will be beneficial in the long term.

\section{CONCLUSION}

A more profound knowledge on SARS-CoV-2 biology and pathophysiology and evolving genome still is necessary to provide novel drugs to modify the course of COVID-19. It will also provide real-time information on the emergence of variants of concern leading to updating currently available vaccines.

\section{REFERENCES}

1. Kofler W, Glazache OS, Lyshol H, Tellnes G. Is fighting against Covid-19 enough? Scand J Pubic Health. 2021;49:9-13.

2. Walls AC, Park YJ, Tortorici MA, Wall A, McGuire AT, Veesler D. Structure, function, and antigenicity of the SARS-CoV-2 spike glycoprotein. Cell. 2020;181:281-92.

3. Wrapp D, Wang N, Corbett KS, Goldsmith JA, Hsieh CL, Abiona O, et al. Cryo-EM structure of the 2019-nCoV spike in the prefusion conformation. Science. 2020;367:1260-3.

4. Ortega JT, Serrano ML, Pujol FH, Rangel HR. Role of changes in SARS-CoV-2 spike protein in the interaction with the human ACE2 receptor: an in-silico analysis. EXCLI J. 2020;19:410-7.

5. Kam YW, Okumura Y, Kido H, Ng LF, Bruzzone R, Altmeyer R. Cleavage of the SARS coronavirus spike glycoprotein by airway proteases enhances virus entry into human bronchial epithelial cells in vitro. PLoS One. 2009;4:e7870.

6. Elrashdy F, Redwan EM, Uversky VN. Why COVID-19 transmission is more efficient and aggressive than viral transmission in previous coronavirus epidemics? Biomolecules. 2020;10:1312.

7. Altan-Bonnet N. Extracellular vesicles are the Trojan horses of viral infection. Curr Opin Microbiol. 2016;32:77-81.

8. Elrashdy F, Aljaddawi AA, Redwan EM, Uversky VN. On the potential role of exosomes in the COVID-19 reinfection/reactivation opportunity. J Biomol Struct Dyn. 2020;2020:1-12.

9. Guilger-Casagrande M, de Barros CT, Antunes VA, de Araujo DR, Lima R. Perspectives and challenges in the fight against COVID-19: the role of genetic variability. Front Cell Infect Microbiol. 2021;11:598875.

10. Kumar A, Prasoon P, Sekhawat PS, Pareek V, Faiq MA, Kumari C, et al. Pathogenesis guided therapeutic management of COVID-19: an immunological perspective. Int Rev Immunol. 2021; 40:54-71.

11. Klann K, Bojkova D, Tascher G, Ciesek S, Muench C, Cinatl ]. Growth factor receptor signaling inhibition prevents SARSCoV-2 replication. Mol Cell. 2020;80:164-74.e4
12. Viswanathan T, Arya S, Chan SH, Qi S, Dai N, Hromas RA, et al. Structural basis of RNA cap modification by SARS-CoV-2. Nat Commun. 2020;11:3718.

13. Sosa-Hernández VA, Torres-Ruiz ], Cervantes-Díaz R, RomeroRamírez S, Páez-Franco JC, Meza-Sánchez DE, et al. B cell subsets as severity-associated signatures in COVID-19 patients. Front Immunol. 2020;11:611004.

14. Del Valle DM, Kim-Schulze S, Huang HH, Beckmann ND, Nirenberg $\mathrm{S}$, Wang $\mathrm{B}$, et al. An inflammatory cytokine signature predicts COVID-19 severity and survival. Nat Med. 2020;26:1636-43.

15. Salama C, Han J, Yau L, Reiss WG, Kramer B, Neidhart JD, et al. Tocilizumab in patients hospitalized with Covid-19 pneumonia. N Engl J Med. 2021;384:20-30.

16. WHO Solidarity Trial Consortium. Repurposed antiviral drugs for Covid-19-interim WHO solidarity trial results. N Engl J Med. 2021;384:497-511.

17. Kalil AC, Patterson TF, Mehta AK, Tomashek KM, Wolfe CR Ghazaryan V, et al, ACCT-2 Study Group Members. Baricitinib plus remdesivir for hospitalized adults with Covid-19. N Engl ] Med. 2021;384:795-807.

18. Dhakal B, Hari PN, Usmani SZ, Hamadani M. Chimeric antigen receptor $\mathrm{T}$ cell therapy in multiple myeloma: promise and challenges. Bone Marrow Transpl. 2021;56:9-19.

19. Bakowski MA, Beutler N, Wolff KC, Kirkpatrick MG, Chen E Nguyen TH, et al. Drug repurposing screens identify chemical entities for the development of COVID-19 interventions. Nat Commun. 2021;12:3309.

20. Cox RM, Wolf JD, Plemper RK. Therapeutically administered ribonucleoside analogue MK-4482/EIDD-2801 blocks SARSCoV-2 transmission in ferrets. Nat Microbiol. 2021;6:11-8.

21. Mei X, Lee HC, Diao KY, Huang M, Lin B, Liu C, et al. Artificial intelligence-enabled rapid diagnosis of patients with COVID-19. Nat Med. 2020;26:1224-8.

22. Bello-Chavolla OY, Antonio-Villa NE, Ortiz-Brizuela E, VargasVázquez A, González-Lara MF, Ponce-de-León A, et al. Validation and repurposing of the MSL-COVID-19 score for prediction of severe COVID-19 using simple clinical predictors in a triage setting: the nutri-CoV score. PLoS One. 2020;15:e0244051.

23. Tregoning JS, Brown ES, Cheeseman HM, Flight KE, Higham SL, Lemm NM, et al. Vaccines for COVID-19. Clin Exp Immunol. 2020;202:162-92.

24. Hodgson SH, Mansatta K, Mallett G, Harris V, Emary KR, Pollard AJ. What defines an efficacious COVID-19 vaccine? A review of the challenges assessing the clinical efficacy of vaccines against SARS-CoV-2. Lancet Infect Dis. 2021;21:e26-35

25. Dai L, Gao GF. Viral targets for vaccines against COVID-19. Nat Rev Immunol. 2021;21:73-82.

26. Konings F, Perkins MD, Kuhn JH, Pallen MJ, Alm EJ, Archer BN, et al. SARS-CoV-2 variants of interest and concern naming scheme conducive for global resources. Nat Microbiol. 2021; 6:821-3.

27. World Health Organization. SARS-CoV-2 Genomic Sequencing for Public Health Goals: interim Guidance. Geneva: World Health Organization; 2021. Available from: https://www.apps.who.int/ iris/handle/10665/338483

28. Harvey WT, Carabelli AM, Jackson B, Gupta RK, Thomson EC Harrison EM, et al. SARS-CoV-2 variants, spike mutations and immune escape. Nat Rev Microbiol. 2021;19:409-24.

29. Campbell F, Archer B, Laurenson-Schafer H, Jinnai Y, Konings F Batra $\mathrm{N}$, et al. Increased transmissibility and global spread of SARS-CoV-2 variants of concern as at June 2021. Euro Surveill. 2021;26:2100509.

30. Lopez-Bernal J, Andrews N, Gower C, Gallagher E, Simmons R, Thelwall S, et al. Effectiveness of COVID-19 vaccines against the B.1.617.2. MedRxiv. 2021 [Epub ahead of print] 